$\$=$

\title{
A collective review of cases with cryptorchidism managed in a teaching hospital in Sudan
}

\author{
Awad Ali M. Alawad*, Walaa Amin Hussain, Faisal H. Younis \\ Department of Surgery, Faculty of Medicine, University of Medical Sciences and Technology, Sudan \\ *Corresponding author E-mail:awadali82@hotmail.com
}

\begin{abstract}
Background: Cryptorchidism is one of the most common urogenital abnormalities in male children. Delayed diagnosis and treatment of cryptorchidism lead to complications such as infertility, malignancy and testis torsion.

Objective: To determine the cause of delayed presentation, complications recorded, the surgical treatment offered in a developing country.

Methods:A retrospective review of patients' records from December 2012 to December 2014 was carried out. Sixty one patients presented to the University Charity Teaching Hospital during this period.

Results: Sixty one patients were included in the study. Age ranged from 1-75 years with mean at surgery is 11 years. Twenty seven patients (44\%) presented after 24 months, $3(4.9 \%)$ less than 12 months, and $23(37.3 \%)$ less than 1 month. Age at operation, $7(13 \%)$ were $\leq 2$ years, $15(28 \%)$ were 2-5years, $39(59 \%)$ were $>5$ years.Fifty four $(88.5 \%)$ patients have undergone orchidopexy, 3(4.9\%) orchidectomies, $1(1.6 \%)$ biopsy, 1 (1.6\%) exploratory laparotomy, and in $2(3.3 \%)$ cases operation wasn't done.

Conclusion:Late presentation observed in this study is alarming and action should be taken by spreading awareness in the community and ensuring adequate neonatal and children examination in order to avoid the complications.
\end{abstract}

Keywords:Undescended Testis; Urogenital Abnormalities; Delayed Diagnosis.

\section{Introduction}

The word cryptorchidism is obtained from the Greek word "Kryptos" meaning "hidden" and "orchid" meaning "testicles" (Khatwa $\&$ Menon 2000). It is one of the most common urogenital abnormalities in newborn boys and very variable figures on its incidence have been described in different type of studies. Several studies have described risk factors such as low birth weight, being born as small for gestational age, and preterm delivery as concerning newborns (Esposito et al. 2008, Khatwa \& Menon 2000, Rubenwolf \& Stein 2013).

The management of undescended testis (UDT) in children poses a serious challenge to the urologist, in terms of management, which depends on how early the patient present. Regardless of age; UDT represents a true problem in Africa as it and its management is challenged by older presentations, due to economic issue, illiteracy, or negligence, lack of laparoscopic equipment and skills, few specialized pediatric centers, and difficult follow-up. Given its high incidence and the few African researches, particularly in Sudan where there is nothing available in the literature about undescended testes, are reasons necessitating this study? The aim of this research is to determine the cause of delayed presentation; complications recorded, the surgical treatment offered in a developing country.

\section{Methods}

A cross-sectional descriptive study was conducted from December 2012 to December 2014 at University Charity Teaching Hospital, affiliated to University of Medical Sciences and Technology (UMST), Khartoum, Sudan.

All patients of undescended testes who presented at this period were included in the study. Primary data was collected through a questionnaire from medical records. The collected data were computerized and statistically analyzed using SPSS (Statistical Package for Social Science) version 19. Descriptive statistics were used to quantify the categorical variables. Chi square was calculated to compare between gender role in specialties and factors affecting specialty choices. $\mathrm{P}$ value $(<0.05)$ was considered significant difference. The ethical approval for this study was obtained from the ethical committee at UMST. An informed consent was obtained from the patients.

\section{Results}

Total number of patients was 61 . Age ranged from 1-75 years with mean at surgery is 11 years. Regarding the side of undescended testes; 28 (45.9\%) were right, 21 (34.4\%) were left, and 12 $(19.7 \%)$ cases had bilateral UDT. There was an obvious variation in the presentation where $27(44 \%)$ cases presented after 24 months, 3 (4.9\%) cases less than 12 months, and 23 (37.3\%) cases less than 1 month. In forty eight $(78.7 \%)$ of patients, the condition was noticed by their parents, $11(18 \%)$ by the patient himself, school camp and patients brother in individual cases representing $(1.6 \%)$ each. Table 1 showed the clinical presentation of the patients.

Regarding the management of these patients, orchidopexy was the treatment of choice. Fifty four $(88.5 \%)$ patients have undergone orchidopexy for either unilateral or bilateral UDT, 3(4.9\%) orchi- 
dectomies, 1 (1.6\%) biopsy, 1 (1.6\%) exploratory laparotomy, and in $2(3.3 \%)$ cases operation wasn't done due to low haemoglobin (Tab 2). Of these patients only 7 (13\%) were $\leq 2 \mathrm{yr}, 15$ (28\%) were $\leq 5 \mathrm{yr}$, while $32(59 \%)$ were $>5 \mathrm{yrs}$. Age ranged from 1-75 years with mean age at surgery 11 to 12 years. Site of the testes as recorded intra-operatively $44(72.1 \%)$ inguinal canal, $6(9.8 \%)$ intraabdominal, $3(4.9 \%)$ not found, $1(1.6 \%)$ at external ring, $1(1.6 \%)$ peritoneal cavity, 1 (1.6\%) under deep ring, and $5(8.2 \%)$ not reported (Fig 1).
The size of the operated testis could not be determined in 58 $(95.1 \%)$ due to lack of data and $3(4.9 \%)$ were of normal size as found by ultrasonography. Complications mainly took the form of 15 inguinal hernias, 3 hydrocele, 2 testicular torsion, and 2 testicular tumors. Five associated anomalies were found in our patients 2 (3.3\%) phimosis, 1(1.6\%) meatal stenosis, 1(1.6\%) hypospadias, $1(1.6 \%)$ micropenis. No records about post operational growth or any follow-up were available.

Table 1: Shows the Clinical Presentation of 61 Patient

\begin{tabular}{lll}
\hline & Frequency & Percentage \\
\hline Empty scrotum & 45 & $74 \%$ \\
Anomalies & 1 & $1.6 \%$ \\
Mass & 11 & $18 \%$ \\
Pain & 3 & $4.9 \%$ \\
Infertility & 1 & $1.6 \%$ \\
\hline
\end{tabular}

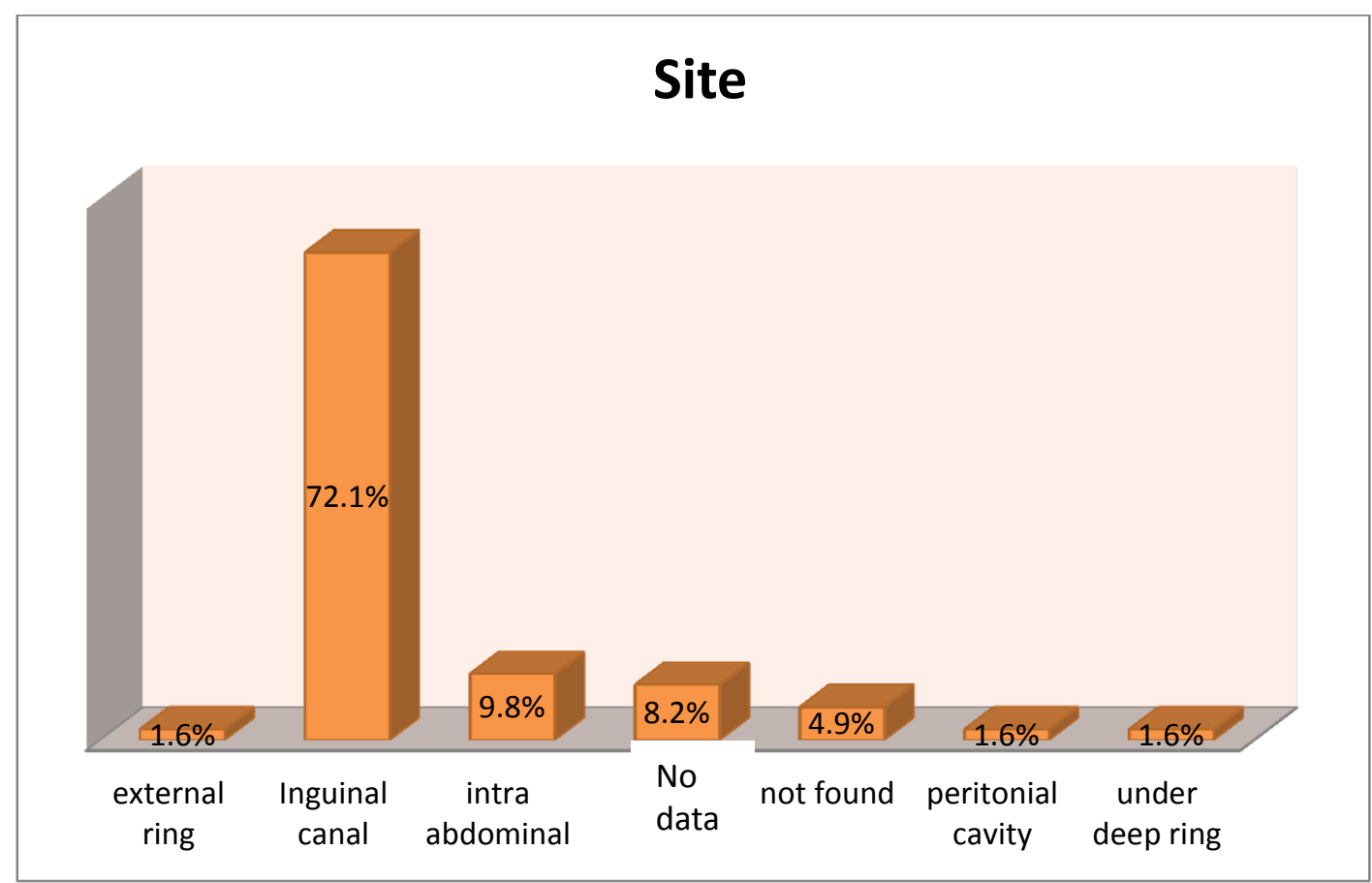

Fig. 1: Shows the Level of Descent and Gross Appearance of 61 Undescended Testes

Table 2: Show the Type of Management Offered To 61 Patients with Undescended Testes

\begin{tabular}{lllll}
\hline & Frequency & Percent & Valid Percent & Cumulative Percent \\
\hline Biopsy & 1 & 1.63 & 1.6 & 1.6 \\
op not done & 2 & 3.3 & 3.3 & 4.9 \\
orchidectomy & 3 & 4.9 & 4.9 & 9.8 \\
orchidopexy & 54 & 88.5 & 88.5 & 98.4 \\
Exploratory laprotomy & 1 & 1.63 & 1.6 & 100 \\
Total & 61 & 100.0 & 100.0 & \\
\hline
\end{tabular}

\section{Discussion}

Undescended testis is a condition of great concern to paediatric surgeons and urologists owing to the risk of impaired spermatogenesis and consequent infertility later in life. The risk of testicular cancer may be higher in these patients as noted by one report in which ten per cent of patients with testicular cancer gave a history of cryptorchidism(Shalaby 2012) even though there appears to be no conclusive proof that correction of cryptorchidism protects against cancer, but renders the test is easily accessible.

We noticed that $45.9 \%$ of cases were right sided. This right side dominance came in consensus with most of the studies reviewed except for one held at Nigeria by David et al. stating that the left side was more affected with $34(47.9 \%)$ involved in unilateral cases than right side 22 (31.0\%) (David \& Iyekoretin 2008), which is contrary to expectation as the left testis is usually lower than the right.
In this study, the condition was noticed by the parents in 48 case $(78.7 \%)$, more than that in study done by Gasana et al. in Tanzania were only in 13 cases $(43.3 \%)$ the condition was discovered by the parent (Osifo \& Osaigbovo 2009) which is quite promising taking in account the level of parental education obtained by questionnaire where $5 / 10$ mothers attained high level education and only $3 / 10$ fathers achieved this level. In 11 cases (18\%), UDT was discovered by the patient himself, school camp and patients siblingin individual cases representing $(1.6 \%)$ each, compared to $9(30 \%)$ cases noticed by the patient, and by health care staff in eight cases $(26.7 \%)$ in Tanzania (Mlay \& Sayi 1994).

In this study, the site of the UDT was inguinal in $72 \%$. The UDT was atrophic in $26.7 \%$ of cases overall. A study from Dar- Salaam reported that $80 \%$ of UDT were inguinal and $29.8 \%$ were atrophied (Mlay \& Sayi 1994). A study from Nigeria reported testicular atrophy in $28 \%$ of patients with UDT (Adeoti et al. 2004). In contrast, another study from Nigeria found atrophy in $60.7 \%$ of UDT in adults, confirming the well-known fact that testicular 
atrophy is associated with delayed surgery (David \& Iyekoretin 2008). In this study, 16 patients $(53.3 \%)$ had associated malformations, of them 13 (43.3\% of the total group of 30) had associated hernia sacs. In a study from Nigeria associated hernia sacs were found in 52\% (Sowande et al. 2015).

To avoid the risk of testicular atrophy and infertility, it is now widely agreed that orchidopexy be performed at age $<2$ years (Ali et al. 2014). In the present study, $13 \%$ of patients had orchidopexy before two years of age, but $60 \%$ had surgery after five years, by which time significant morphological changes would have occurred in the testis. A study in Nigeria found that $11 \%$ of patients with UDT had orchidopexy before two years, $42 \%$ before five years and 58\% after five years of age (David \& Iyekoretin 2008). A study from Tanzania reported that $50 \%$ of patients presented for orchidopexy after five years of age. Another study from Nigeria reported correction of UDT in 52.2\% of patients after five years and in $26.9 \%$ who presented as adults (Adeoti et al. 2004). A recent study from Ireland reported that only $29 \%$ of patients with UDT proceeded to surgery before two years, and the mean age at orchidopexy was 5.6 years (Sowande et al. 2015). In contrast, a study from the USA reported that the median age at consultation and surgery was 20.3 and 28.9 months, respectively (Mlay \& Sayi 1994). In our environment, the current median age at surgery is 11 years; the reasons for this delay include, late presentation, ignorance of initial attending doctors regarding age at optimal treatment and lack of recognition by midwives. To improve on the age at surgery, it is necessary to educate parents, midwives and doctors as suggested before.

Orchidopexy represents the gold standard treatment for UDT. Advances in laparoscopic surgery have led to laparoscopy being used by most pediatric surgeons as both a diagnostic and therapeutic tool. In our setting, laparoscopy is not available. In this study, orchidopexy was the only mode of treatment available where 54( $88.5 \%$ ) patient have undergone orchidopexy for either unilateral or bilateral UDT,3(4.9\%) orchidectomies, 1 (1.6\%) biopsy, 1 (1.6\%) exploratory laparotomy,and in $2(3.3 \%)$ cases operation was not done due to low haemoglobin.

\section{Conclusion}

The late presentation detected in this study is alarming, because the majority of patients were diagnosed and treated after two years of age.Health-care workers should perform neonatal examination to detect UDT and inform parents that early correction of UDT will decrease the risk of infertility and facilitate future examination to detect the development of testicular malignancy.

\section{References}

[1] Adeoti ML, Fadiora SO, Oguntola AS, Aderounmu AO, Laosebikan DA \& Adejumobi OO (2004): Cryptorchidism in a local population in Nigeria. West Afr $J$ Med 23, 62-64. http://dx.doi.org/10.4314/wajm.v23i1.28085.

[2] Ali HAM, Alawad A, Ali AS \& Abdalla AAE (2014): Pattern of developmental sex disorders in a tertiary hospital in Sudan. International Journal of Medicine 2, 53-55. http://dx.doi.org/10.14419/ijm.v2i2.2957.

[3] David OO \& Iyekoretin E (2008): Undescended testes in a developing country: a study of the management of 71 patients. Afr J Paediatr Surg 5, 11-14. http://dx.doi.org/10.4103/0189-6725.41629.

[4] Esposito C, Caldamone AA, Settimi A \& El-Ghoneimi A (2008): Management of boys with nonpalpable undescended testis. Nat Clin Pract Urol 5, 252-260. http://dx.doi.org/10.1038/ncpuro1102.

[5] Khatwa UA \& Menon PS (2000): Management of undescended testis Indian J Pediatr 67, 449-454. http://dx.doi.org/10.1007/BF02859466.

[6] Mlay SM \& Sayi EN (1994): Undescended testis in paediatric patients at Muhimbili Medical Centre, Dar es Salaam. East Afr Med J 71, 135137.

[7] Osifo DO \& Osaigbovo EO (2009): The prevalence, postnatal descent, and complications of undescended testes among children who underwent neonatal circumcision in Benin City, Nigeria. J Pediatr Surg 44, 791-796. http://dx.doi.org/10.1016/i.jpedsurg.2008.06.009.
[8] Rubenwolf P \& Stein R (2013): [Diagnosis and management of the undescended testis--an update in the light of the current guidelines]. Aktuelle Urol 44, 445-451. http://dx.doi.org/10.1055/s-0033-1358664.

[9] Shalaby MM (2012): Is there a role for open surgery in the management of the undescended testis? Afr J Paediatr Surg 9, 97. http://dx.doi.org/10.4103/0189-6725.99390.

[10] Sowande OA, Talabi AO, Etonyeaku AC \& Adejuyigbe O (2015): Groin exploration for the nonpalpable testes: a single center experience. Niger J Surg 21, 56-59. http://dx.doi.org/10.4103/11176806.153195. 